\title{
On the Minimum Degree of Minimal Ramsey Graphs
}

\author{
Tibor Szabó \\ McGill University \\ szabo@math.mcgill.ca
}

\author{
Philipp Zumstein \\ ETH Zürich \\ zuphilip@inf.ethz.ch
}

\section{Stefanie Zürcher}

ETH Zürich

zuerchers@student.ethz.ch

June 17, 2009

\begin{abstract}
We investigate the minimization problem of the minimum degree of minimal Ramsey graphs, initiated by Burr, Erdős, and Lovász. We determine the corresponding graph parameter for numerous bipartite graphs, including bi-regular bipartite graphs and forests. We also make initial progress for graphs of larger chromatic number. Numerous interesting problems remain open.
\end{abstract}

\section{Introduction}

A graph $G$ is called $H$-Ramsey, denoted by $G \rightarrow H$, if in every edge-coloring of $G$ with colors red and blue there is a monochromatic $H$. Furthermore, if every proper subgraph of an $H$-Ramsey graph $G$ is not $H$-Ramsey, then we say that $G$ is $H$-minimal. We denote the family of all $H$-minimal graphs by $\mathcal{M}(H)$. The classical theorem of Ramsey states that for all graphs $H$ the family $\mathcal{M}(H)$ is nonempty.

A significant portion of Ramsey theory is concerned with finding the extremal value of various graph parameters over the family $\mathcal{M}(H)$. The most widely investigated among these questions is the minimization of the number of vertices $n(G)$ over all graphs $G \in \mathcal{M}(H)$. The quantity $\min _{G \in \mathcal{M}(H)} n(G)$ is the classical Ramsey number $r(H)$ of $H$. For a regularly updated survey on Ramsey numbers of all kinds of graphs, see [10].

Another natural parameter is the size Ramsey number $\hat{r}(H)$ which is the minimum of the edge number $e(G)$ over all graphs in $G \in \mathcal{M}(H)$. The size Ramsey number was introduced by Erdős, Faudree, Rousseau, and Schelp in [4] and studied further extensively by many others (see [6] for a recent survey).

In the present paper we are interested in the quantity

$$
s(H):=\min _{G \in \mathcal{M}(H)} \delta(G)
$$


where $\delta(G)$ is the minimum degree of the graph $G$. This parameter was introduced and first studied by Burr, Erdős, and Lovász [1]. By the minimality condition one cannot just simply add vertices of small degree to an $H$-Ramsey graph and get thereby a small upper bound for $s(H)$. It is rather the case that each of the vertices, in particular a vertex of minimum degree, has to be important to produce a monochromatic copy of $H$. This leads to the following lower bound.

Lemma 1.1 ("simple lower bound", [7]). For all graphs $H$

$$
s(H) \geq 2 \delta(H)-1 .
$$

For a proof, consider a graph $G$ with $\delta(G)<2 \delta(H)-1$ and let $v \in V(G)$ be of degree at most $2 \delta(H)-2$. If there exists an edge-coloring of $G-v$ without monochromatic $H$, we can extend this coloring to an edge-coloring of $G$ by coloring at most $\delta(G)-1$ of the edges incident to $v$ red and the remaining at most $\delta(G)-1$ edges blue. The coloring of $G$ obtained this way does not contain a monochromatic $H$, which implies that $G$ cannot be $H$-minimal.

A clique of order $r(H)$ is $H$-Ramsey, but maybe it is not $H$-minimal. Since the minimum degree of any subgraph of the clique is not larger than the minimum degree of the clique itself, we have $s(H) \leq r(H)-1$. The determination of $r\left(K_{k}\right)$ is out of reach currently and is one of the most notorious open problems in combinatorics. In a striking contrast, $s\left(K_{k}\right)$ turned out to be more approachable and was computed exactly for every $k$ by Burr et. al [1,2]: they obtained that $s\left(K_{k}\right)=(k-1)^{2}$. An alternative proof was found by Fox and Lin [7].

Observe that the simple lower bound $2 \delta(H)-1$ is far from being tight for the clique. However, Fox and Lin [7] showed that it is tight for all complete bipartite graphs $K_{a, b}$, i.e., $s\left(K_{a, b}\right)=2 \min \{a, b\}-1$. They also raised the question whether the simple lower bound (1) would be tight for any other graph or rather, for which graphs it is tight.

In the present paper we prove that the simple lower bound is tight for a large class of bipartite graphs, including paths, even cycles, and more generally, all trees and all bi-regular bipartite graphs.

\section{$1.1 \quad$ Notation}

Let $G, H$ be two graphs and assume that $V(G) \cap V(H)=\emptyset$. Then $G+H$ denotes the disjoint sum of $G$ and $H$, i.e., $V(G+H)=V(G) \cup V(H), E(G+H)=$ $E(G) \cup E(H)$. Furthermore, $t H$ denotes the disjoint sum of $t$ copies of $H$. The join $G \vee H$ is the graph obtained from $G+H$ by adding all the edges $\{x, y\}$ where $x \in V(G)$ and $y \in V(H)$.

We say that there is a copy of $H$ in $G$ if there is an injective map $\varphi$ : $V(H) \rightarrow V(G)$ such that if $\left\{h_{1}, h_{2}\right\} \in E(H)$ then also $\left\{\varphi\left(h_{1}\right), \varphi\left(h_{2}\right)\right\} \in E(G)$. An injective map $\varphi: V(H) \rightarrow V(G)$ such that $\left\{h_{1}, h_{2}\right\} \in E(H)$ if and only if $\left\{\varphi\left(h_{1}\right), \varphi\left(h_{2}\right)\right\} \in E(G)$ is called an (induced) embedding of $H$ in $G$.

For $A, B \subseteq V(G)$ let $E(A, B)$ denote the set of edges with one endpoint in $A$ and the other in $B$. For $A=B$, we abbreviate by $E(A):=E(A, A)$. We write $G[A]$ for the induced subgraph of $G$ spanned by the vertices of $A$. The neighborhood of a vertex $x$ is denoted by $N(x):=\{y \in V(G):\{x, y\} \in E(G)\}$ and the degree of $x$ by $\operatorname{deg}(x):=|N(x)|$. 
A bipartition $(A, B)$ of a bipartite graph $H$ is a partition of the vertices $V(H)=A \cup B$ such that $E(A, B)=E(H)$. If $H$ is connected, then there is only one bipartition. We define the parameters $a(H)$ and $b(H)$ by

$$
a(H):=\min \{|S|:(S, V(H) \backslash S) \text { is a bipartition }\},
$$

and $b(H):=n(H)-a(H)$. For a bipartite graph $H$ with bipartition $(A, B)$ let $\Delta_{A}(H)\left(\Delta_{B}(H)\right)$ be the largest among the degrees of vertices in $A(B)$. A bipartite graph $H$ is called bi-regular if there is a bipartition $(A, B)$ such that $\operatorname{deg}(x)=\Delta_{A}(H)$ for every $x \in A$ and $\operatorname{deg}(x)=\Delta_{B}(H)$ for every $x \in B$.

We use the notation $[n]:=\{1,2, \ldots, n\}$ and the set of all $k$-element subsets of a set $S$ is denoted by $\left(\begin{array}{l}S \\ k\end{array}\right)$. A c-coloring of a set $S$ is a function from $S$ to a $c$-element set, most often to $[c]$. The $k$-uniform hypergraph Ramsey number $r_{k}\left(a_{1}, \ldots, a_{c}\right)$ is the smallest number $n \in \mathbb{N}$ such that in every $c$-coloring of $\left(\begin{array}{c}{[n]} \\ k\end{array}\right)$ there is a color $i \in[c]$ and a subset $A \subseteq[n]$ of $a_{i}$ elements such that all the $k$-subsets of $A$ are colored with the color $i$. We write $r_{k}(a)$ if all $a_{i}$ are equal to $a$ and for $k=2$ we omit the index and just write $r\left(a_{1}, \ldots, a_{c}\right)$.

\section{$1.2 \quad$ Results}

Isolated vertices do not pose any further restriction on edge-colorings but they may increase the order of the underlying graph. We handle them with the following proposition.

Proposition 1.2. Let $H$ be a graph without isolated vertices and for some $t \geq 1$ define $H^{\prime}=H+t K_{1}$.

(i) If $t>r(H)-n(H)$ then $s\left(H^{\prime}\right)=0$.

(ii) If $t \leq r(H)-n(H)$ then $s\left(H^{\prime}\right)=s(H)$.

Proof. (i) There exists a graph $K$ on exactly $r(H)$ vertices that is $H$-minimal. Then the graph $K+(t-r(H)+n(H)) K_{1}$ is clearly $H^{\prime}$-minimal and has minimum degree 0 .

(ii) We claim that a graph $G$ is $H$-Ramsey if and only if it is $H^{\prime}$-Ramsey. This in turn implies that a graph is $H$-minimal if and only if it is $H^{\prime}$-minimal and the proposition follows.

If $G$ is $H$-Ramsey then, by the definition of $r(H), n(G) \geq r(H)$. Hence in any edge-coloring of $E(G)$ there are at least $r(H)-n(H)$ vertices besides a monochromatic copy of $H$ to accommodate the $t$ isolated vertices of $H^{\prime}$.

As a consequence of the above proposition, we can restrict our attention to graphs with minimum degree at least 1 .

Theorem 1.3. Let $H$ be a bipartite graph with $\delta(H) \geq 1$ and assume that there exists a bipartition $(A, B)$ of $H$ such that $|\{v \in B: \operatorname{deg}(v)>\delta(H)\}| \leq a(H)-1$. Then

$$
s(H)=2 \delta(H)-1 .
$$

We note that our theorem implies the result of Fox and Lin [7] that $s\left(K_{a, b}\right)=$ $2 \min \{a, b\}-1$ for every $a \geq 1, b \geq 1$, but our construction is different from theirs.

Corollary 1.4. (i) For all paths $P_{k}, k \geq 2$, we have $s\left(P_{k}\right)=1$. 
(ii) For all even cycles $C_{2 k}, k \geq 2$, we have $s\left(C_{2 k}\right)=3$.

(iii) For all bi-regular bipartite graphs $H$ with $\delta(H) \geq 1$, we have $s(H)=$ $2 \delta(H)-1$.

(iv) For all connected bipartite graphs $H=(A \cup B, E)$ with $|A|=|B|$ we have $s(H)=2 \delta(H)-1$.

(v) For every tree $T$, we have $s(T)=1$.

Define $\mathcal{G}_{\delta}$ to be the family of bipartite graphs $H$ with $\delta(H)=\delta$ for which there is a bipartition $(A, B)$ such that $|\{v \in B: \operatorname{deg}(v)>\delta(H)\}| \leq a(H)-1$. Theorem 1.3 states that for each graph in $\mathcal{G}_{\delta}$ we have $s(H)=2 \delta-1$.

Observation. If $H_{1} \in \mathcal{G}_{\delta}$ and $\delta\left(H_{2}\right) \geq \delta$ then $H_{1}+H_{2} \in \mathcal{G}_{\delta}$.

Let $\left(A_{1}, B_{1}\right)$ be a good bipartition of $H_{1}$ and let $\left(A_{2}, B_{2}\right)$ be a bipartition of $H_{2}$ such that $\left|B_{2}\right|=a\left(H_{2}\right)$. We have $a\left(H_{1}+H_{2}\right)=a\left(H_{1}\right)+a\left(H_{2}\right)$ and it is easy to see that $\left(A_{1} \cup A_{2}, B_{1} \cup B_{2}\right)$ is a good bipartition of $H_{1}+H_{2}$.

The following corollary is an immediate consequence of the above observation and Corollary 1.4.

Corollary 1.5. (i) For all forests $F$ with $\delta(F)=1$, we have $s(F)=1$.

(ii) For all bipartite graphs $H$ with $1 \leq \delta(H) \leq \Delta(H) \leq 2$, we have $s(H)=$ $2 \delta(H)-1$.

Indeed, the graphs in (ii) are disjoint sums of paths and even cycles.

The smallest bipartite graph for which we do not know the value of the function $s$ can be interpreted as a $K_{2,2}$ with an edge $K_{2}$ "hanging" at one of its vertices (see Figure 1.2). The minimum degree of this graph is 1 , which gives

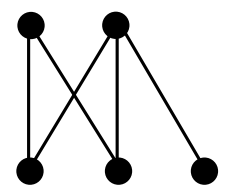

Figure 1: $1 \leq s\left(K_{2,2} \cdot K_{2}\right) \leq 3$

1 as a "simple lower bound" for its $s$-value. One feels though that the effect of the $K_{2}$ hanging from the $K_{2,2}$ might not be so great and the answer rather should be closer to the $s$-value of $K_{2,2}$, which is 3 . This motivates our following investigation about how a small structure (a $K_{2}$ ) attached in various ways to a big structure (a $K_{t}$ ) can effect the $s$-value of the graph. Not surprisingly, we find that the effect is less and less.

In the following theorem we consider the disjoint union of a $t$-clique and an edge. While this graph has minimum degree 1 , its $s$ value grows quadratically in $t$. In particular, the "simple lower bound" can be arbitrarily bad for $\delta(H)=1$.

Theorem 1.6.

$$
s\left(K_{t}+K_{2}\right)= \begin{cases}1, & \text { for } t=2,3 \\ (t-1)^{2}, & \text { for } t \geq 4\end{cases}
$$


The second part of Theorem 1.6 is a consequence of something much stronger: For $t \geq 4$,

$$
G \rightarrow K_{t} \Leftrightarrow G \rightarrow K_{t}+K_{2}
$$

Let $K_{t} \cdot K_{2}$ denote the graph which contains a complete graph on $t$ vertices and one additional vertex connected to exactly one vertex of the $t$-clique. We call this additional edge and additional vertex a hanging edge and a hanging vertex, respectively. The minimum degree of $K_{t} \cdot K_{2}$ is 1 . By Corollary 1.4 we see that the simple lower bound $s\left(K_{t} \cdot K_{2}\right) \geq 1$ is sharp for $t=1$ and $t=2$. We prove that these are the only two cases when this happens.

Theorem 1.7. For every $t \geq 3$,

$$
s\left(K_{t} \cdot K_{2}\right) \geq t-1 .
$$

This lower bound is tight for $t=3$.

\section{Proposition 1.8.}

$$
s\left(K_{3} \cdot K_{2}\right)=2 .
$$

Theorem 1.3 and Corollary 1.4 are proved in the next section. In Section 3 we establish Theorem 1.6, while the proofs of Theorem 1.7 and Proposition 1.8 are presented in Section 4. Finally, in Section 5 we discuss some open problems.

\section{Bipartite Graphs}

Recall that $a(H):=\min \{|S|:(S, V(H) \backslash S)$ is a bipartition $\}$ and $b(H):=$ $n(H)-a(H)$. For all graphs $H$ with $a(H)=a, b(H)=b$ we obviously have $H \subseteq K_{a, b}$, but $H \nsubseteq K_{a-1, m}$ for all positive integers $m$.

\subsection{Balanced Colorings}

Lemma 2.1. Let $H$ be a bipartite graph with $a(H)=a$. Then for all positive integers $m$

$$
K_{2 a-2, m} \nrightarrow H .
$$

Proof. Let us denote by $V$ the partite set of $K_{2 a-2, m}$ having size $2 a-2$. We partition $V$ into two sets $V_{1}$ and $V_{2}$, each of size $a-1$, and we color the edges incident to $V_{1}$ red and the edges incident to $V_{2}$ blue. This edge-coloring does not contain a monochromatic $H$, since the red and blue graphs are each copies of $K_{a-1, m} \nsupseteq H$.

The edge-coloring in the above proof has the property that both monochromatic subgraphs are copies of $K_{a-1, m}$. We call such an edge-coloring of $K_{2 a-2, m}$ balanced. Next, we show that if an edge-coloring of $K_{2 a-2, m}$ has no monochromatic copy of $H$ then it contains a balanced coloring of $K_{2 a-2, d}$, provided $m$ is large enough.

Lemma 2.2. Let $H$ be a bipartite graph with $a(H)=a$ and $b(H)=b$ and let $d$ be an integer. Then there exists an integer $m=m(a, b, d)$ such that in every red/blue edge-coloring of $K_{2 a-2, m}$ there exists

(i) a monochromatic copy of $H$, or 
(ii) a copy of $K_{2 a-2, d}$ with a balanced coloring .

Proof. It is enough to prove the lemma for $d \geq b$ because a copy of $K_{2 a-2, d}$ with a balanced coloring contains a copy of $K_{2 a-2, d^{\prime}}$ with a balanced coloring for $d^{\prime} \leq d$. Set $m:=(d-1) \cdot 2^{2 a-2}+1$ and denote by $V$ and $W$ the partite sets of $K_{2 a-2, m}$ of size $2 a-2$ and $m$, respectively. Consider an arbitrary edge-coloring $c$ of $K_{2 a-2, m}$ with colors red and blue. Let $v_{1}, v_{2}, \ldots, v_{2 a-2}$ be the elements of $V$ in some ordering. Assign for each vertex $w \in W$ a vector $p(w) \in\{\text { red, blue }\}^{2 a-2}$ such that $p(w)_{i}=c\left(\left\{w, v_{i}\right\}\right)$ for $i=1,2, \ldots, 2 a-2$. There are $2^{2 a-2}$ possible $p$ vectors. By the pigeonhole principle there exists at least $d$ vertices $w_{1}, \ldots w_{d} \in$ $W$ with the same $p$-vector. If the number of red entries in $p\left(w_{1}\right)$ is at least $a$, then the vertices $w_{1}, \ldots, w_{d}$ and $a$ of the vertices of $V$ corresponding to the red entries of $p\left(w_{1}\right)$ form a monochromatic red copy of $K_{a, d} \supseteq K_{a, b} \supseteq H$. The case of at least $a$ blue entries in $p\left(w_{1}\right)$ is analogous. Otherwise, $p\left(w_{1}\right)$ has $a-1$ red and $a-1$ blue entries, meaning that the vertices $w_{1}, \ldots, w_{d}$ and $V$ induce a $K_{2 a-2, d}$ with a balanced coloring.

\subsection{The construction}

Let $m, k \in \mathbb{N}$. We define the bipartite incidence graph $S(m, k)=(A \cup B, E)$ by

$$
A=\{1,2, \ldots, m\}, \quad B=\left(\begin{array}{l}
A \\
k
\end{array}\right), \quad E=\{\{a, T\}: T \in B, a \in T\} .
$$

Lemma 2.3 (Nešetřil, Rödl [9]; cf. Diestel [3] p.264). Let $H$ be a bipartite graph.

(i) There exist integers $m, k$ such that $H$ can be embedded into $S(m, k)$. In fact, we can choose $k=a(H)+1$.

(ii) For every $k, m \in \mathbb{N}$ there exists integer $m^{\prime}$ such that

$$
S\left(m^{\prime}, 2 k-1\right) \rightarrow S(m, k) .
$$

Corollary 2.4. For every bipartite graph $H=(A \cup B, E)$ we have

$$
s(H) \leq 2 a(H)+1 .
$$

We will modify the above lemma using a slightly different construction and thereby improve the bound on $k$. We will then apply this to derive our main theorem.

Definition 2.5. Let $G$ be a graph, $J \subseteq V(G)$, and $k \geq 2, \ell \geq 1$. Then we define $\mathcal{T}_{k}^{\ell}(G ; J)$ to be the graph $\left(V^{\prime}, E^{\prime}\right)$ with

$$
\begin{aligned}
& V^{\prime}=V(G) \cup\left(\left(\begin{array}{l}
J \\
k
\end{array}\right) \times[\ell]\right), \\
& E^{\prime}=E(G) \cup\left\{\{x,(M, i)\}: M \in\left(\begin{array}{l}
J \\
k
\end{array}\right), x \in M, i \in[\ell]\right\} .
\end{aligned}
$$

The graph defined above can be obtained from $G$ by first designating a subset $J$ of the vertices of $G$ and then for each $k$-tuple $M$ of $J$ adding $\ell$ new distinct vertices and connecting them to all vertices in $M$. It is clear, that $\left|V^{\prime}\right|=$ $|V(G)|+\left(\begin{array}{c}|J| \\ k\end{array}\right) \cdot \ell,\left|E^{\prime}\right|=|E(G)|+\left(\begin{array}{c}|J| \\ k\end{array}\right) \cdot \ell \cdot k$. Furthermore note that unless $|J|<k$, the degree of all new introduced vertices is $k$. Observe that $\mathcal{T}_{k}^{1}\left(E_{n} ;[n]\right)=S(n, k)$ for $E_{n}$ being the empty graph on the vertices $[n]=\{1,2, \ldots, n\}$. 
Lemma 2.6. Let $H=(A \cup B, E)$ be bipartite.

(i) There exist integers $n, k, \ell$ such that $H$ can be embedded in $\mathcal{T}_{k}^{\ell}\left(E_{n},[n]\right)$. In fact, we can choose $k=\Delta_{B}(H)$ and map $A$ into $V\left(E_{n}\right)$.

(ii) For every $n, k, \ell$ there exists $n^{\prime}, \ell^{\prime}$, with the property that

$$
\mathcal{T}_{2 k-1}^{\ell^{\prime}}\left(E_{n^{\prime}},\left[n^{\prime}\right]\right) \rightarrow \mathcal{T}_{k}^{\ell}\left(E_{n},[n]\right),
$$

such that the image of $V\left(E_{n}\right)$ from the monochromatic copy of $\mathcal{T}_{k}^{\ell}\left(E_{n},[n]\right)$ is contained in $V\left(E_{n^{\prime}}\right)$ of $\mathcal{T}_{2 k-1}^{\ell^{\prime}}\left(E_{n^{\prime}},\left[n^{\prime}\right]\right)$.

Proof. (i) Set $n=|A|+\Delta_{B}(H), k=\Delta_{B}(H), \ell=|B|$. In order to find an embedding $\varphi: H \rightarrow \mathcal{T}_{k}^{\ell}\left(E_{n},[n]\right)$, first arbitrarily map $A$ onto $[|A|]$ then process the vertices of $B$ in an arbitrary order: For each $w \in B$ it holds that $|N(w)| \leq k$, so we can choose $L=\varphi(N(w)) \cup\{|A|+1, \ldots,|A|+(k-\operatorname{deg}(w))\} \in\left(\begin{array}{c}{[n]} \\ k\end{array}\right)$ and map $w$ to $(L, i)$ for some unused $i$ (there is at least one unused $i$ by the definition of $\ell$ ).

(ii) Set $\ell^{\prime}=2\left(\begin{array}{c}2 k-1 \\ k\end{array}\right)(\ell-1)+1, n^{\prime}=r_{k}(n, n, 2 k-1)$ (the $k$-uniform hypergraph Ramsey number for three colors) and let $K=\mathcal{T}_{2 k-1}^{\ell^{\prime}}\left(E_{n^{\prime}} ;\left[n^{\prime}\right]\right)$. Color the edges of $K$ with red and blue. The degree of each vertex $(M, i) \in V(K) \backslash\left[n^{\prime}\right]$ is $2 k-1$, so there is a color $c_{M, i}$ which appears at least $k$ times among the edges incident to $(M, i)$. Hence we can define a function $\varphi:\left(\begin{array}{c}{\left[n^{\prime}\right]} \\ 2 k-1\end{array}\right) \times\left[\ell^{\prime}\right] \rightarrow\{$ red, blue $\} \times\left(\begin{array}{c}{\left[\begin{array}{c}n^{\prime} \\ k\end{array}\right)} \\ \text { such }\end{array}\right.$ that all edges of $K$ between $(M, i)$ and the second component $(\varphi(M, i))_{2}$ (which is a $k$-element subset of $M)$ is colored with the first component $(\varphi(M, i))_{1}$. For any fixed $M \in\left(\begin{array}{c}{\left[n^{\prime}\right]} \\ 2 k-1\end{array}\right)$, there are $2\left(\begin{array}{c}2 k-1 \\ k\end{array}\right)$ many possible $\varphi$-values. Thus, by the definition of $\ell^{\prime}$ and the pigeonhole principle, at least $\ell$ of the vertices from $\left\{(M, 1),(M, 2), \ldots,\left(M, \ell^{\prime}\right)\right\}$ have the same $\varphi$-value; let us denote this value by $\varphi_{M}$.

We now define an auxiliary coloring of the $k$-tuples $\left(\begin{array}{c}{\left[n^{\prime}\right]} \\ k\end{array}\right)$. For a subset $S \in\left(\begin{array}{c}{\left[n^{\prime}\right]} \\ k\end{array}\right)$, if there exists an $M \in\left(\begin{array}{c}{\left[n^{\prime}\right]} \\ 2 k-1\end{array}\right)$ such that $\left(\varphi_{M}\right)_{2}=S$ then $S$ receives the color $\left(\varphi_{M}\right)_{1}$ (if there are more than one such $M$ then we choose one of them arbitrarily). This way we obtain a partial red/blue coloring of $\left(\begin{array}{c}{\left[n^{\prime}\right]} \\ k\end{array}\right)$ which we extend by giving each yet uncolored $k$-tuple the color white. By the choice of $n^{\prime}$ there is

(a) a set of size $n$ with only red $k$-tuples or

(b) a set of size $n$ with only blue $k$-tuples or

(c) a set of size $2 k-1$ with only white $k$-tuples.

Case (c) does not occur because by definition, every $2 k-1$ tuple $M \in\left(\begin{array}{c}{\left[n^{\prime}\right]} \\ 2 k-1\end{array}\right)$ does contain a red or a blue $k$-tuple, namely $\left(\varphi_{M}\right)_{2}$.

The cases (a) and (b) are symmetric, therefore we can assume that we have a set $A^{\prime} \subseteq\left[n^{\prime}\right]$ of size $n$ containing only red $k$-tuples of the auxiliary coloring. This means that for each $k$-tuple $T \subseteq A^{\prime}$, there is a $(2 k-1)$-set $M_{T} \supseteq T$ such that $\left(\varphi_{M_{T}}\right)_{2}=T$ and $\left(\varphi_{M_{T}}\right)_{1}=$ red. Hence there are $\ell$ vertices of the form $\left(M_{T}, i\right)$ each of which has only red edges towards $T$. In particular there is a red copy of $\mathcal{T}_{k}^{\ell}\left(E_{n} ;[n]\right)$ in $K$.

By part (i) and (ii) of Lemma 2.6, we have the following corollary.

Corollary 2.7. For every bipartite graph $H=(A \cup B, E)$

$$
s(H) \leq 2 \min \left\{\Delta_{A}(H), \Delta_{B}(H)\right\}-1 .
$$


Proof of Theorem 1.3. Let $(A, B)$ be a bipartition of $H$ with $\mid\{v \in B: \operatorname{deg}(v)>$ $\delta(H)\} \mid \leq a(H)-1$. Let $S \subseteq\{v \in B: \operatorname{deg}(v)=\delta(H)\}$ be an arbitrary subset such that $|B \backslash S|=a(H)-1=: a^{\prime}$. Clearly $S \neq \emptyset$ because there is no bipartition where one part is smaller than $a(H)$. Let $N(S) \subseteq A$ denote the set of vertices adjacent to at least one vertex in $S$. The graph $H^{*}=H[S \cup N(S)]$ has a bipartition, namely $(S, N(S))$, such that $\operatorname{deg}(s)=\delta(H), \forall s \in S$, i.e., $\Delta_{S}\left(H^{*}\right)=\delta(H)$. According to Lemma 2.6 there exist integers $n=n\left(H^{*}\right)$ and $\ell=\ell\left(H^{*}\right)$ with the property that

$$
\mathcal{T}_{2 \delta(H)-1}^{\ell}\left(E_{n} ;[n]\right) \rightarrow H[S \cup N(S)],
$$

such that in the monochromatic copy of $H[S \cup N(S)]$ the set $N(S)$ is contained in $V\left(E_{n}\right)$. Without loss of generality we can assume that $n \geq|A|$.

By Lemma 2.2, there is an integer $m=m(a(H), b(H), n)$ such that in every edge-coloring of $G=K_{2 a^{\prime}, m}$ there exists a monochromatic $H$ or there is a copy of $K_{2 a^{\prime}, n}$ with a balanced coloring. Let $L$ and $M$ be the partite sets of $G$ with size $2 a^{\prime}$ and $m$, respectively. Now we show that

$$
\mathcal{T}_{2 \delta(H)-1}^{\ell}(G ; M) \rightarrow H
$$

Let $c$ be an arbitrary red/blue edge-coloring of $\mathcal{T}_{2 \delta(H)-1}^{\ell}(G ; M)$. The restriction of $c$ to $E(G)$ either contains a monochromatic $H$ and we are done, or otherwise there is a copy $K$ of $K_{2 a^{\prime}, n}$ with a balanced coloring. Let $L$ and $M^{\prime} \subseteq M$, $\left|M^{\prime}\right|=n$, be the partite sets inducing $K$.

Consider $\mathcal{T}_{2 \delta(H)-1}^{\ell}\left(E_{n} ; M^{\prime}\right)$ which is certainly a subgraph of $\mathcal{T}_{2 \delta(H)-1}^{\ell}(G ; M)$. By (3) there exists a monochromatic, say blue, copy $T$ of $H[S \cup N(S)]$, such that the image of $N(S)$ is contained in $M^{\prime}$. Since $\left|M^{\prime}\right| \geq|A|$ we have space to embed the vertices of $A \backslash N(S)$ in $M^{\prime} \backslash V(T)$. Hence the union of $T$ and the blue copy of $K_{a^{\prime}, n}$ in $K$ contains a blue copy of $H$ and (4) follows.

On the other hand by Lemma $2.1 G \nrightarrow H$, and hence there is an $H$-minimal graph $G^{\prime}$, such that $G \subseteq G^{\prime} \subseteq \mathcal{T}_{2 \delta(H)-1}^{\ell}(G ; M)$. The minimum degree of $G^{\prime}$ is clearly at most $2 \delta(H)-1$ and the theorem follows.

Proof of Corollary 1.4. Parts $(i)-(i v)$ are immediate. For $(v)$, let $X$ and $Y$ be the partite sets of the tree $T$. We can easily apply Theorem 1.3 unless $|X| \neq|Y|$ and all vertices of minimum degree are contained in the larger of the two partite sets.

Hence assume that $|X|>|Y|=a(T)$ and the set of all vertices of degree 1 , denoted by $S$, is contained in $X$. To apply Theorem 1.3 it is enough to show that $|X \backslash S|<|Y|$. Fix an arbitrary vertex $r \in Y$ as the root of the tree and define the successor relation according to it. All vertices in $X \backslash S$ have at least one successor in $Y$ and these all have to be different (because there are no cycles). Thus the function succ : $X \backslash S \rightarrow Y$ is injective. Since the root vertex $r$ is not the successor of any vertex, we have

$$
|Y| \geq|\operatorname{succ}(X \backslash S)|+1 \geq|X \backslash S|+1 .
$$




\section{Complete Graph with Disjoint Edge}

Proof of Theorem 1.6. By Lemma 1.1 we have $s\left(K_{r}+K_{2}\right) \geq 1$ for all $r \geq 2$. A matching of size three shows that $s\left(K_{2}+K_{2}\right)=1$. For $r=3$, we claim that $K_{6}+K_{2}$ is $\left(K_{3}+K_{2}\right)$-minimal, and thus $s\left(K_{3}+K_{2}\right)=1$.

In any red/blue edge-coloring of $K_{6}$ there is at least one monochromatic $K_{3}$. To avoid a monochromatic $K_{3}+K_{2}$ the edge-coloring must contain two vertex disjoint monochromatic copies of $K_{3}$ : one in blue and one in red. No matter how we color the extra edge, we will get a monochromatic $K_{3}+K_{2}$, i.e., $K_{6}+K_{2} \rightarrow K_{3}+K_{2}$.

The graph $K_{6}$ minus one edge has an edge-coloring without a monochromatic $K_{3}$, and $K_{6}$ is not $\left(K_{3}+K_{2}\right)$-Ramsey: The coloring consisting of a red $K_{4}$ and all remaining edges blue contains no monochromatic $K_{3}+K_{2}$. Hence $K_{6}+K_{2}$ is $\left(K_{3}+K_{2}\right)$-minimal and $s\left(K_{3}+K_{2}\right)=\delta\left(K_{6}+K_{2}\right)=1$.

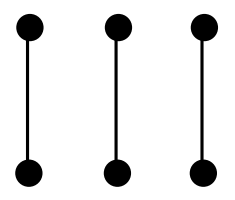

(a) $s\left(K_{2}+K_{2}\right)=1$

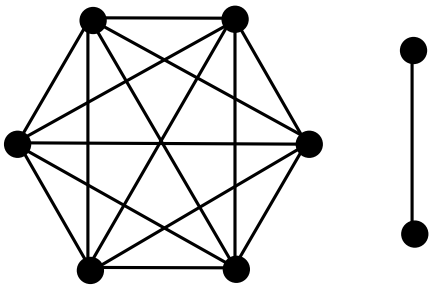

(b) $s\left(K_{3}+K_{2}\right)=1$

Figure 2: Minimal graphs

For $t \geq 4$ we prove that a graph is $K_{t}$-Ramsey if and only if it is $\left(K_{t}+\right.$ $K_{2}$ )-Ramsey. We are thankful to the anonymous referee who pointed out the following stronger statement (and its consequences in Corollary 5.2).

Theorem 3.1. Let $a_{1} \geq a_{2} \geq \ldots \geq a_{s} \geq 1$ and define $H_{i}:=K_{a_{1}}+\ldots+K_{a_{i}}$ for $1 \leq i \leq s$. If $r\left(a_{1}, a_{1}-a_{s}+1\right)>2\left(a_{1}+\ldots+a_{s-1}\right)$, then $G \rightarrow H_{s}$ if and only if $G \rightarrow H_{s-1}$.

To conclude (2) we can apply Theorem 3.1 with $s=2, a_{1}=t$, and $a_{2}=2$, and use that $r(t, t-1)>2 t$ for $t \geq 4$. This finishes the proof of Theorem 1.6 and it remains to prove Theorem 3.1.

Proof of Theorem 3.1. Since $H_{s-1}$ is a subgraph of $H_{s}$, if $G \rightarrow H_{s}$ then also $G \rightarrow H_{s-1}$. Thus it suffices to show that $G \rightarrow H_{s-1}$ implies $G \rightarrow H_{s}$.

Let $G$ be a graph such that $G \rightarrow H_{s-1}$ and suppose for contradiction that $G \nrightarrow H_{s}$. Let $c$ be a red/blue edge-coloring of $G$ without monochromatic $H_{s}$. Without loss of generality, we may assume that there is a blue copy of $H_{s-1}$, and let $S_{1}$ be its vertex set. Since $c$ has no blue $H_{s}$, the coloring restricted to $V(G) \backslash S_{1}$ has no blue $K_{a_{s}}$. Define $H_{0}$ to be the empty graph. Let $i$ be the largest index such that $V(G) \backslash S_{1}$ contains a red $H_{i}$ and let $S_{2}$ be its vertex vertex set (it may happen that $S_{2}$ is empty). Since $c$ has no red $H_{s}$, we have $i<s$. The coloring $c$ restricted to $V(G) \backslash\left(S_{1} \cup S_{2}\right)$ contains no red $K_{a_{1}}$.

Our goal is now to recolor some of the edges of $G$ such that the resulting coloring $c^{\prime}$ contains no monochromatic $K_{a_{1}}$. We have $\left|S_{1} \cup S_{2}\right|=\left|V\left(H_{s-1}\right)\right|+$ 
$\left|V\left(H_{i}\right)\right| \leq 2\left(a_{1}+\ldots+a_{s-1}\right)<r\left(a_{1}, a_{1}-a_{s}+1\right)$ so by the definition of the Ramsey number we can recolor the edges inside $S_{1} \cup S_{2}$ such that there is no red $K_{a_{1}}$ and no blue $K_{a_{1}-a_{s}+1}$. All edges between $S_{1} \cup S_{2}$ and $V(G) \backslash\left(S_{1} \cup S_{2}\right)$ are recolored to blue, while the color of the other edges does not change. The largest blue clique restricted to $S_{1} \cup S_{2}$ has at most $a_{1}-a_{s}$ vertices, the largest blue clique in $V(G) \backslash\left(S_{1} \cup S_{2}\right)$ has at most $a_{s}-1$ vertices, which implies that $c^{\prime}$ contains no blue copy of $K_{a_{1}}$. Since there are no red edges between $S_{1} \cup S_{2}$ and $V(G) \backslash\left(S_{1} \cup S_{2}\right)$, the largest red clique contains less than $a_{1}$ vertices. Therefore there is no monochromatic $K_{a_{1}}$ in $c^{\prime}$. This is a contradiction to $G \rightarrow H_{s-1}$ and the proof is complete.

We discuss further consequences of Theorem 3.1 in Section 5 .

\section{Complete Graph with Hanging Edge}

To prove Theorem 1.7 we introduce some auxiliary notation and lemmas. Let us assume that we are given a graph $H$ with some red/blue edge-coloring $c$ without a monochromatic $K_{t} \cdot K_{2}$ and assume from now on that $t \geq 3$. We call a vertex which is contained in two monochromatic copies of $K_{t}$ critical, a vertex which is contained in one monochromatic copy of $K_{t}$ harmless, and other vertices safe. For a vertex $u \in V(H)$, we denote the set of those neighbors of $u$ which are adjacent to $u$ via a red (blue) edge by $R(u)(B(u))$.

Lemma 4.1. Let $u \in V(H)$ be a critical vertex. Then

(i) $|R(u)|=|B(u)|=t-1$, i.e., $u$ is contained in exactly one red and exactly one blue copy of $K_{t}$ and has no other incident edges.

(ii) $E(R(u), B(u))=\emptyset$, i.e., there is no edge between the red neighbors of $u$ and the blue neighbors of $u$.

Proof. ( $i$ ) Since $u$ is critical it has to be incident to two monochromatic $K_{t}$. If they would be in the same color then this would yield a monochromatic $K_{t} \cdot K_{2}$. A red $K_{t}$ and a blue $K_{t}$ can only share one vertex. Also, there cannot be more edges incident to $u$ without creating a monochromatic $K_{t} \cdot K_{2}$.

(ii) Assume there is an edge between a red neighbor of $u$ and a blue neighbor of $u$. Then this edge has some color according to $c$ and therefore it completes a monochromatic $K_{t} \cdot K_{2}$ in this color with one of the $t$-cliques containing $u$.

Lemma 4.2. For every graph $H$ and every edge-coloring $c$ without a monochromatic $K_{t} \cdot K_{2}$ there exists a new edge-coloring $c_{F}$ with no critical vertices and no monochromatic $K_{t} \cdot K_{2}$.

Proof. For each $t$-clique which is monochromatic in $c$ and has a critical vertex, choose one arbitrary edge containing a critical vertex. Let us denote the set of these edges by $F \subseteq E(H)$ and let $c_{F}$ be the coloring after we change the color of each edge in $F$.

By Lemma 4.1, we see that every critical vertex $u$ is incident to exactly two $t$-cliques and none of them is monochromatic in the coloring $c_{F}$, since we changed the color of exactly one edge in each. Thus each critical vertex in $c$ is safe in $c_{F}$.

Every edge whose color was changed contains a critical vertex in $c$, which is safe in $c_{F}$, meaning that these edges cannot be part of a new monochromatic 


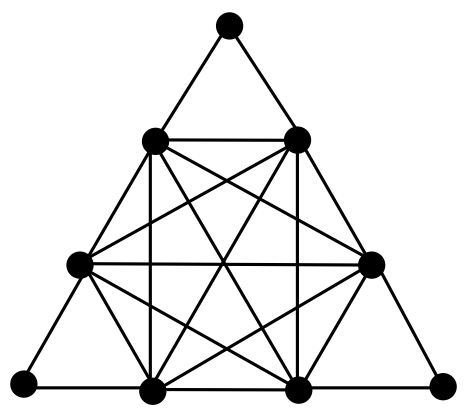

Figure 3: $s\left(K_{3} \cdot K_{2}\right) \leq 2$

$K_{t}$. That is, every monochromatic $K_{t}$ in $c_{F}$ was already monochromatic in $c$ and hence no vertices are critical in $c_{F}$.

We still need to show that there is no monochromatic $K_{t} \cdot K_{2}$ in $c_{F}$. Since every monochromatic $K_{t}$ in $c_{F}$ was monochromatic in $c$, and $c$ has no monochromatic $K_{t} \cdot K_{2}$, the only possibility to have a monochromatic $K_{t} \cdot K_{2}$ in $c_{F}$ would be that the hanging edge $e$ changed its color. Let $U$ be the vertex set of the $K_{t}$ within a monochromatic, say blue, $K_{t} \cdot K_{2}$ in $c_{F}$. Then $U$ was already blue in $c$, while $e$ was red in $c$. Since $e$ changed its color, it was part of a red $t$-clique $W$ in $c$. Hence $U \cap W=\{u\}$, where $u$ is an endpoint of $e$, and $u$ is critical in $c$. This is a contradiction, since $u$ is not safe in $c_{F}$.

Proof of Theorem 1.7. Suppose for contradiction that there is a $K_{t} \cdot K_{2}$-minimal graph $\mathrm{G}$ with a vertex $x$ of degree less than $t-1$. Since $G-x$ is not $K_{t} \cdot K_{2}$ Ramsey, there is a red/blue edge-coloring $c$ of $G-x$ without a monochromatic $K_{t} \cdot K_{2}$. By Lemma 4.2 we can also assume that $c$ has no critical vertices. We now extend $c$ to a coloring of $G$. Color each edge $\{x, y\}$ of $G$ red if $y$ is contained in a blue $t$-clique of $c$ and blue otherwise. Since there are no critical vertices, $y$ cannot be contained in both a blue and a red $t$-clique, therefore the definition of the coloring extension is well-defined.

Since $x$ has degree less than $t-1$, it can contribute to a monochromatic $K_{t} \cdot K_{2}$ only as a hanging vertex. Let $e$ be the hanging edge of a monochromatic $K_{t} \cdot K_{2}$ containing $x$ and let $U$ be the monochromatic $t$-clique. By the definition of the extended coloring, the color of the edge $e$ and $U$ is different, a contradiction.

Proof of Proposition 1.8. By Theorem 1.7 we know that $s\left(K_{3} \cdot K_{2}\right) \geq 2$. Therefore it is enough to give a $K_{3} \cdot K_{2}$-minimal graph $G$ with minimum degree 2.

We extend $K_{6}$ by three paths of length 2 , see Figure 3 , and claim that this graph $G$ is $K_{3} \cdot K_{2}$-minimal. In any red/blue edge-coloring of $K_{6}$ there is a monochromatic triangle. It is possible to color $K_{6}$ without a monochromatic $K_{3} \cdot K_{2}$, namely coloring two disjoint triangles blue and coloring all other edges by red. It is easy to see that, up to renaming of the vertices and the colors, this is the only such edge-coloring. In any partition of the $K_{6}$ of $G$ into two triangles $T_{1}, T_{2}$, there is a path $P$ of length 2 connecting a vertex in $T_{1}$ and a vertex in $T_{2}$. Suppose the edges of $T_{1}$ and $T_{2}$ are colored blue. Then both edges of the path $P$ are colored red. Hence $P$ and the other red edges going between $T_{1}$ and $T_{2}$ yield a red $K_{3} \cdot K_{2}$. This shows that the graph $G$ is $K_{3} \cdot K_{2}$-Ramsey. 
If we delete an edge from the $K_{6}$ then we can color $K_{6}$ without monochromatic triangles and extend this to each of the three 2-paths by coloring their two edges with distinct colors. If we delete an edge $e$ lying on a 2-path of $G$ then it is easy to color $G-e$ without creating a monochromatic $K_{3} \cdot K_{2}$ : partition the vertex set of the $K_{6}$ of $G$ into two blue triangles such that each of the endpoints of the remaining two 2-path are completely contained in one of the blue triangles and color all other edges red. It is easy to see that this edge-coloring has no monochromatic $K_{3} \cdot K_{2}$. Hence $G$ is $\left(K_{3} \cdot K_{2}\right)$-minimal.

\section{$5 \quad$ Remarks and Open Problems}

The following concept arises naturally from Proposition 1.2 and Theorem 3.1.

Definition 5.1. Two graphs $H$ and $K$ are called Ramsey-equivalent if the set of $H$-Ramsey graphs and the set of $K$-Ramsey graphs are the same. Otherwise, $H$ and $K$ are called Ramsey-separable.

Clearly, the relation of being Ramsey-equivalent is an equivalence relation. Furthermore, if $H$ and $K$ are Ramsey-equivalent then the set of $H$-minimal and $K$-minimal graphs are the same and $s(H)=s(K), r(H)=r(K), \hat{r}(K)=\hat{r}(H)$.

By repeatedly applying Theorem 3.1 one obtains part $(i)$ of the following corollary, while part $(i i)$ is an immediate consequence of part $(i)$.

Corollary 5.2. (i) Let $a_{1} \geq \ldots \geq a_{s} \geq 1$ be such that $r\left(a_{1}, a_{1}-a_{i}+1\right)>$ $2\left(a_{1}+\ldots+a_{i-1}\right)$ for all $i=2, \ldots, s$. Then $K_{a_{1}}+\ldots+K_{a_{s}}$ is Ramseyequivalent to $K_{a_{1}}$.

(ii) The graph $K_{t}+s K_{k}$ is Ramsey-equivalent to $K_{t}$ for $k \leq t-2$ and $s<\frac{r(t, t-k+1)-2(t-k)}{2 k}$.

For the two extremes of the spectrum of $k$, we spell out the concrete bounds by substituting known results into part $(i i)$.

(a) $K_{t}+s K_{t-2}$ is Ramsey-equivalent to $K_{t}$ for some $s=\Omega\left(\frac{t}{\log t}\right)$,

(b) $K_{t}+s K_{2}$ is Ramsey-equivalent to $K_{t}$ for $t \geq 4$ and some $s=\Omega\left(t 2^{t / 2}\right)$.

For $(a)$, one uses that $r(t, 3)=\Omega\left(\frac{t^{2}}{\log t}\right)$ proven by Kim [8], for $(b)$ one can use $r(t, t-1)=\Omega\left(t 2^{t / 2}\right)$ proven by Erdős [5].

The following two observations provide Ramsey-separable graphs.

Proposition 5.3. Let $t \geq 1$.

(i) $K_{t}$ and $K_{t}+K_{t}$ are Ramsey-separable.

(ii) $K_{t}$ and $K_{t-1}$ are Ramsey-separable.

Proof. ( $i$ ) Let $R=r\left(K_{t}, K_{t}\right)$ and $G=K_{R}$. Then $G \rightarrow K_{t}$ but $K_{R-1} \nrightarrow K_{t}$. Extend an edge-coloring of $K_{R-1}$ without a monochromatic $K_{t}$ arbitrarily to $K_{R-1} \vee x \cong K_{R}$. All monochromatic $K_{t}$ in this extended coloring have to contain the vertex $x$ and therefore we do not find two vertex-disjoint ones. This proves $G \nrightarrow K_{t}+K_{t}$.

(ii) Nešetřil and Rödl [9] proved that $\min \{\chi(G): G \rightarrow H\}=\chi(H)$. Thus two graphs with different chromatic number are Ramsey-separable, in particular $K_{t}$ and $K_{t-1}$ are Ramsey-separable. 
We cannot confirm the status of the pair $K_{t}$ and $K_{t}+K_{t-1}$; we tend to think they are Ramsey-equivalent, at least for large $t$.

Problem 1.a. Prove that $K_{t}$ and $K_{t}+K_{t-1}$ are Ramsey-equivalent for large enough $t$.

Theorem 3.1 provides many pairs of graphs that are Ramsey-equivalent. Still the structure of these pairs is quite limited: one is always the union of some connected components of the other. We do not have an example of a pair of connected graphs that are Ramsey-equivalent. For example we suspect that the graphs $K_{t}$ and $K_{t} \cdot K_{2}$ are Ramsey-equivalent for large $t$.

Problem 1.b. Prove that $K_{t}$ and $K_{t} \cdot K_{2}$ are Ramsey-equivalent for large enough $t$.

Theorem 1.3 determines the exact value of the graph parameter $s$ for many bipartite graphs. In each of these cases the simple lower bound is tight, which motivates the following question.

Problem 2. Does there exist a bipartite graph $G$ with $s(G)>2 \delta(G)-1$ ?

The smallest unknown case is the graph of $K_{2,2}$ with a hanging edge (see Figure 1.2). For this graph we cannot even decide whether it is Ramsey equivalent to $K_{2,2}$.

We find that the determination of $s(H)$ is also interesting from an algorithmic point of view. At this point it is not even clear to us whether, given a fixed graph $H$, there is an algorithm computing $s(H)$. Should we be able to restrict the search space to a finite subset of $H$-minimal graphs, $s(H)$ would of course be computable. Following [7], we define $S(H)$ to be the minimum positive integer $v$ such that there exists an $H$-minimal graph $G$ with exactly $v$ vertices and $\delta(G)=s(H)$. We would be very much interested in upper bounding $S(H)$ by some, at least modestly reasonable function.

Problem 3. Does there exist a computable function $u$ such that for each graph $H$, we have $S(H) \leq u(H)$ ?

The analogous question for the classical Ramsey number $r(H)$ and the size Ramsey number $\hat{r}(H)$ clearly have a positive answer.

Acknowledgment. We would like to thank the anonymous referees for their thorough work and insightful comments. One of them simplified the proofs of Theorems 1.7 and 3.1 considerably, also correcting an error in the latter.

\section{References}

[1] S. A. Burr, P. Erdős, and L. Lovász. On graphs of Ramsey type. Ars Combinatoria, 1(1):167-190, 1976. 
[2] S. A. Burr, R. J. Faudree, and R. H. Schelp. On Ramsey-minimal graphs. In Proceedings of the Eighth Southeastern Conference on Combinatorics, Graph Theory and Computing (Louisiana State Univ., Baton Rouge, La., 1977), pages 115-124. Congressus Numerantium, No. XIX, Winnipeg, Man., 1977. Utilitas Math.

[3] R. Diestel. Graph theory, volume 173 of Graduate Texts in Mathematics. Springer-Verlag, Berlin, third edition, 2005.

[4] P. Erdős, R. J. Faudree, C. C. Rousseau, and R. H. Schelp. The size ramsey number. Peridoica Mathematica Hungarica, 9(2-2):145-161, 1978.

[5] P. Erdös. Some remarks on the theory of graphs. Bull. Amer. Math. Soc., 53:292-294, 1947.

[6] R. J. Faudree and R. H. Schelp. A survey of results on the size Ramsey number. In Paul Erdös and his mathematics, II (Budapest, 1999), volume 11 of Bolyai Soc. Math. Stud., pages 291-309. János Bolyai Math. Soc., Budapest, 2002.

[7] J. Fox and K. Lin. The minimum degree of ramsey-minimal graphs. Journal of Graph Theory, 54(2):167-177, 2006.

[8] J. H. Kim. The Ramsey number $r(3, t)$ has order of magnitude $t^{2} / \log t$. Random Struct. Algorithms, 7(3):173-207, 1995.

[9] J. Nešetřil and V. Rödl. Simple proof of the existence of restricted Ramsey graphs by means of a partite construction. Combinatorica, 1(2):199-202, 1981.

[10] S. Radziszowski. Small Ramsey numbers. Electronic Journal of Combinatorics. 